\title{
Parametric Study of Changes in Human Balancing Skill by Repeated Balancing Trials on Rolling Balance Board
}

\author{
Csenge A. Molnar ${ }^{1,2^{*}}$, Tamas Insperger ${ }^{1,2}$ \\ ${ }^{1}$ Department of Applied Mechanics, Faculty of Mechanical Engineering, Budapest University of Technology and Economics, \\ 5 Múegyetem rkp., H-1111 Budapest, Hungary \\ 2 MTA-BME Lendület Human Balancing Research Group, 5 Mủegyetem rkp., H-1111 Budapest, Hungary \\ * Corresponding author, e-mail: csenge.molnar@mm.bme.hu
}

Received: 23 March 2020, Accepted: 10 June 2020, Published online: 31 August 2020

\begin{abstract}
Dynamic balance conditions were realized by asking eight volunteers to stand on uniaxial balance board with adjustable geometry and to carry out $60 \mathrm{~s}$ long balancing trials. Four different balance board geometry were used, each associated with different difficulty level. Balancing trials were repeated five times weekly (learning period) in order to test improvement of balancing skill. The measurement was repeated eight weeks after the learning period in order to check the persistence of the balancing skill (confirmation session). Oscillations of ankle angle and hip angle were monitored by OptiTrack motion capture system and four stabilometry parameters were used to characterize improvement in balancing performance, namely, Standard Deviation (STD), Largest Amplitude (LA), Normalized Path Length (NPL) and Mean Power Frequency (MPF). STD and NPL show similar tendency to the preliminary expectations, therefore they can be considered as good measures to describe balancing performance. Results show that subjects used ankle strategy for the less difficult balance board configurations, while for the more difficult tasks, hip strategy was also involved. Changes in STD and NPL during the learning period showed that the improvement and the persistence in balancing skill is more significant for more difficult balancing tasks.

Keywords
\end{abstract}

dynamic balance, stabilometry parameters, balance board, postural sway, human balancing

\section{Introduction}

Number of falls caused by loss of balance is increasing worldwide and poses a serious challenge in the aging societies [1, 2], therefore more and more research effort is devoted to the investigation of human balancing. The main risks of falls are nonactive lifestyle, decreased medical conditions, impaired mobility, cognitive disorders, foot problems, attenuated vision and increased reaction time [3-5]. In order to prevent falls, several interventions can be found in the literature, including modification of living environment, psychological treatment, changes in nutrition and performing balancing exercises [6-8].

A significant part of the ongoing research focuses on the mathematical modeling of the operation of the Central Nervous System (CNS) during balancing tasks. The behavior of the CNS can be analyzed by performing simple balancing tasks that can be described by lowdegree-of-freedom mechanical models. For example, stick balancing [9-11], ankle strategy during quiet standing [12-15] and ball and beam [16] balancing are often modelled as a single-segment single-joint inverted pendulum, while hip strategy during quiet standing [17-19] and standing on a balance board [20-22] are modelled as a system of double inverted pendulum.

Properties, that determine balancing skills of a subject, can be included in the mechanical model, such as flexibility and adaptability of the musculature and the tendons (described by stiffness and damping) [23, 24], proper functioning of the sensory organs (control gains of the controller) [10, 12], and reaction time (feedback delay in the control loop) [25-27]. Analysis of the corresponding mathematical models gives an insight into the effect of the parameter changes on the dynamic behavior of the system. This can help to identify the critical elements of the balancing process and to develop techniques in order to improve the stabilization process.

One of the simplest balancing tasks associated with accidental falls is standing still. Standing still is more challenging on an unstable surface, for example 
on a pinned or rolling balance board [20-22, 28-31]. Balancing abilities can be analyzed under different conditions when subjects are asked to stand on a balance board with adjustable geometry [29]. The purpose of the present study is to analyze how repeated balancing trials performed on rolling balance boards of different geometries redound to the improvement of balancing skill and how the development can be characterized.

\section{Methods}

The goal of the study was to analyze the development of balancing abilities of human subjects on balance boards of different difficulty levels over a 5 -week period. The movement of the human subjects and the balance board was measured in order to determine how ankle or ankle-hip strategies change over the learning period.

\subsection{Participants}

Eight young healthy individuals were involved in the experiment (6 males, 2 females: $27.50 \pm 3.12$ years, $68.13 \pm 10.92 \mathrm{~kg}$, $171.75 \pm 6.20 \mathrm{~cm}$, BMI: 22.96 \pm 2.49 ). Participants did not report any known visual or balance pathology and did not take part in any measurements involving a balance board before. The research was carried out following the principles of the Declaration of Helsinki. Since the within-subject effect of repeated balancing trials was in the focus, subjects were not separated by gender. All participants provided informed consent for all research testing and were given the opportunity to withdraw from the study at any time.

\subsection{Balance board}

A uniaxial rolling balance board was designed and manufactured for the balancing tests, which consists of a board and two wheels as shown in Fig. 1. The elements were

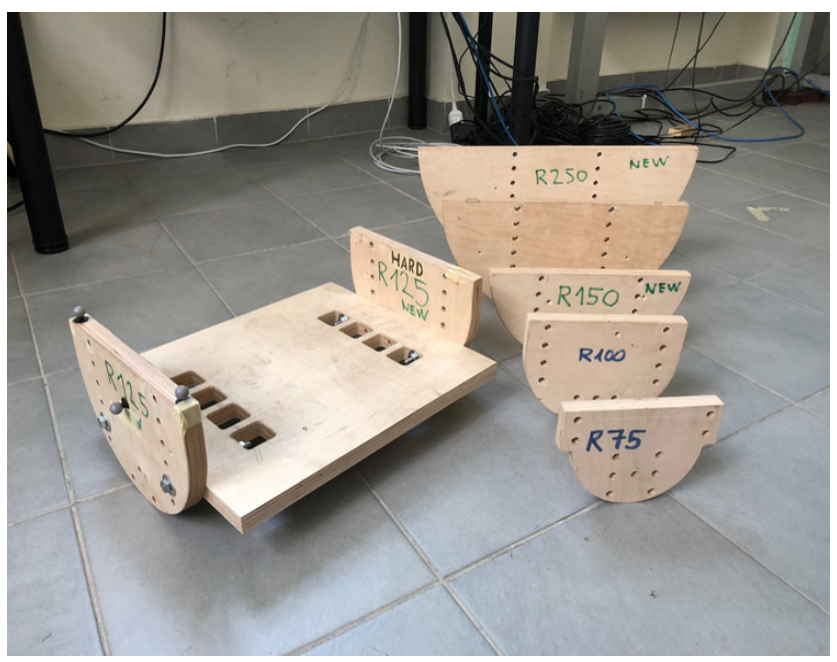

Fig. 1 Balance board with different wheel radii. made from plywood with $21 \mathrm{~mm}$ of thickness and were connected to each other with four screws. Wheels were available with different radius and the elevation of the board was also adjustable.

Preliminary studies showed [29, 31], that the radius of the wheels has a great influence on the balancing performance: the smaller the radius, the more difficult standing on the board. Four different wheel radii were selected among the available sizes for the balancing trials, namely, $125,100,75$ and $50 \mathrm{~mm}$, while the elevation of the board from the ground was the same. The largest and smallest radius was chosen based on the conclusion of previous balancing tests, where all subjects were able to stand on the balance board of radius $125 \mathrm{~mm}$ and nobody was able to balance on the board with radius $50 \mathrm{~mm}$.

For the sake of simplicity, the following terminology is introduced based on the difficulty levels of ski slopes. The learner ski slopes are indicated by green, beginner slope by blue, intermediate by red and expert slopes by black. Following this color code, the balance boards of radius $125 \mathrm{~mm}$ (easiest), $100 \mathrm{~mm}, 75 \mathrm{~mm}$ and $50 \mathrm{~mm}$ (most challenging) were indicated by green, blue, red and black colors, respectively.

\subsection{Experimental procedure}

Each subject participated in five balancing sessions on the same day in five succeeding weeks (learning period). One session consisted of maximum five balancing trials on each balance board starting with the green one. A balancing trial was considered to be successful if the subject was able to balance on the balance board for $60 \mathrm{~s}$ without touching the ground. If one out of the five trials was successful, then the subject was asked to stand on the next balance board (green $\rightarrow$ blue $\rightarrow$ red $\rightarrow$ black). A full balancing session with four balance boards took approximately 20 minutes. Eight weeks later, on the $13^{\text {th }}$ week, all subjects were tested again (confirmation session) in order to check how the balancing skill persisted. In order to provide a safe measurement environment and to prevent the potential accidents, a wooden railing was placed next to the participants (see Fig. 2).

For the standardization of the balancing trials, the following experimental protocol was applied. Subjects were asked to stand on the balance board in socks such that their transverse axis was parallel with the axis of rotation of the board, this way they had to balance in the sagittal plane (anterior-posterior direction). Furthermore, they were instructed to stand in a shoulder width stance with open eyes, stretched legs, look straight ahead at the wall and 


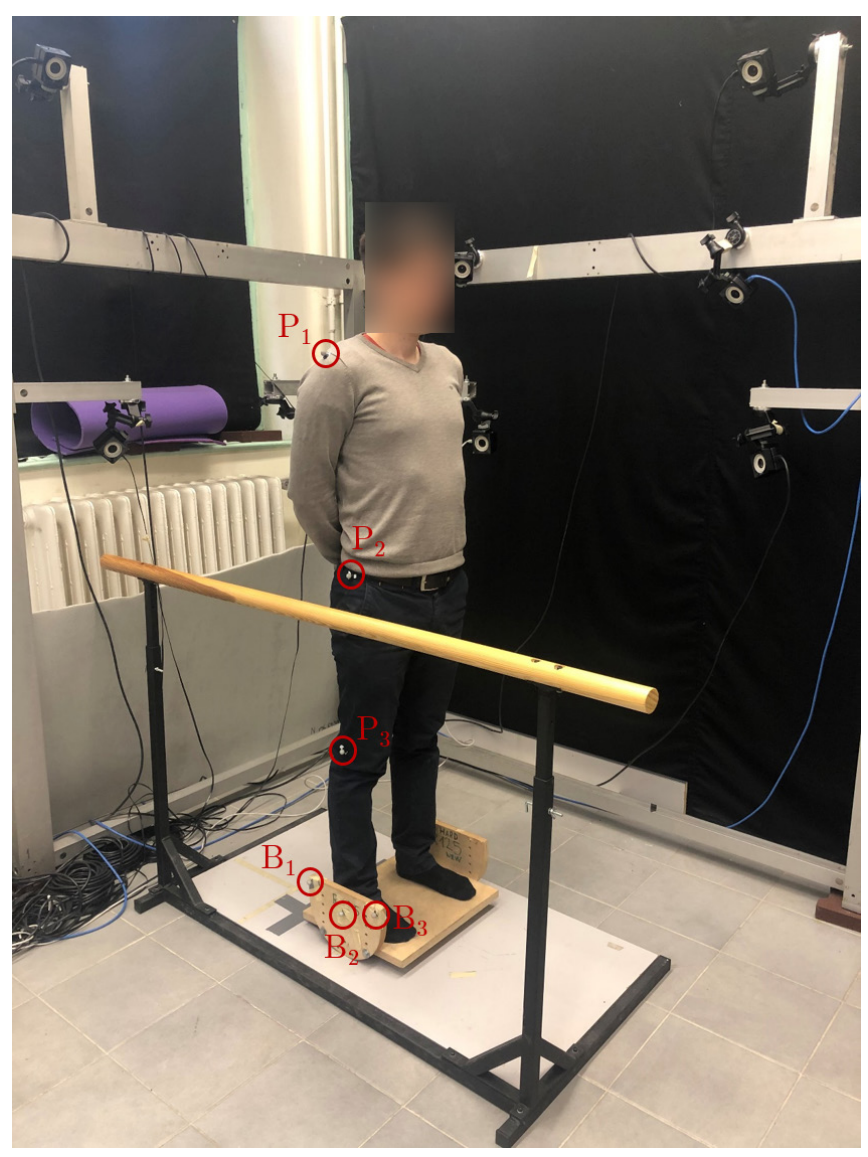

a)

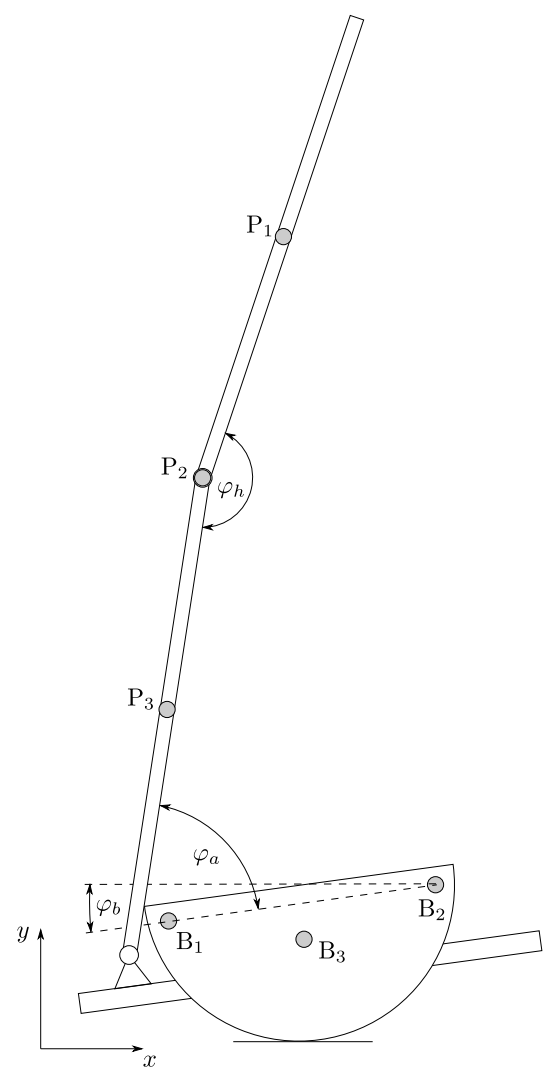

b)

Fig. 2 a) Measurement setup: passive markers placed on the body segments and the balance board.

b) Notation of the markers used during the calculations.

possibly load both legs equally. Moving arms may hide the markers placed on the body segments, therefore hands had to be clasped behind the back to avoid data loss as can be seen in Fig. 2. Participants were asked to balance themselves only using their ankle and to stand on the board as quietly as possible but they were allowed to bend at the hip when it was necessary. Single leg or eyes closed trials were not performed because of increased risk of falling. Abrupt stand up may result about 15 seconds of dizziness due to blood pressure drop [32]. To avoid this effect subject were standing about 3 minutes before the measurement began and they arrived at the measurement in a calm state of mind and relaxed in case of each measurement occasion.

OptiTrack motion capture system was used to record the balancing trials with 8 Prime 13 cameras. The sampling frequency was $120 \mathrm{~Hz}$ satisfying the Shannon sampling theory, since the frequency content of the balance board angle signal above $3 \mathrm{~Hz}$ is considered to be related to noise. Three-three passive markers of diameter $12 \mathrm{~mm}$ were placed on the balance board and the human body, respectively, as can be seen in Fig. 2. Motive software was used to obtain a csv file containing the 3D position of the markers at each time step.

\subsection{Data processing}

Post-processing was performed using Matlab with a self-developed script. Markers placed on the balance board are denoted by $B_{1}, B_{2}$ and $B_{3}$, markers placed on shoulder, hip and knee by $P_{1}, P_{2}$ and $P_{3}$, respectively, as shown in Fig. 2. Spatial position of each marker is interpreted as a vector $\boldsymbol{r}$ pointing from the origin to the specific point. The ankle angle $\varphi_{a}$ is defined as the angle between the line connecting $B_{1}-B_{2}$, and $P_{2}-P_{3}$ as

$\varphi_{a}=\cos ^{-1} \frac{\boldsymbol{r}_{B_{1} B_{2}} \cdot \boldsymbol{r}_{P_{3} P_{2}}}{\left|\boldsymbol{r}_{B_{1} B_{2}}\right| \cdot\left|\boldsymbol{r}_{P_{3} P_{2}}\right|}$,

where

$\boldsymbol{r}_{B_{1} B_{2}}=\boldsymbol{r}_{B_{2}}-\boldsymbol{r}_{B_{1}}$

is the vector pointing from marker $B_{1}$ to $B_{2}$ and 
$\boldsymbol{r}_{P_{3} P_{2}}=\boldsymbol{r}_{P_{2}}-\boldsymbol{r}_{P_{3}}$

is the vector pointing from marker $P_{3}$ to $P_{2}$. The hip angle $\varphi_{h}$ is defined as the angle between the trunk and the lower limbs, therefore the line connecting $P_{2}-P_{1}$, and $P_{2}-P_{3}$ as

$\varphi_{h}=\cos ^{-1} \frac{\boldsymbol{r}_{P_{2} P_{3}} \cdot \boldsymbol{r}_{P_{2} P_{1}}}{\left|\boldsymbol{r}_{P_{2} P_{3}}\right| \cdot\left|\boldsymbol{r}_{P_{2} P_{1}}\right|}$,

where

$\boldsymbol{r}_{P_{2} P_{3}}=\boldsymbol{r}_{P_{3}}-\boldsymbol{r}_{P_{2}}$

is the vector pointing from marker $P_{2}$ to $P_{3}$ and

$\boldsymbol{r}_{P_{2} P_{1}}=\boldsymbol{r}_{P_{1}}-\boldsymbol{r}_{P_{2}}$

The angle of the balance board $\varphi_{b}$ can be determined in the same way.

In case of successful balancing, the measurement was terminated after approximately $65-70 \mathrm{~s}$ in order to provide safely a $60 \mathrm{~s}$ long signal. In case of unsuccessful trials, the length of the signal was equal to the balancing time and the last seconds was associated with the loss of balance. In order to analyze clearly the balancing process, the first $5 \mathrm{~s}$ of the data, as long as subjects find their equilibrium, were removed, and $50 \mathrm{~s}$ sampling interval of the data were used for further calculations. In some cases, when the $60 \mathrm{~s}$ long balancing was not successful, but the subject was able to balance at least for $35 \mathrm{~s}$, time history between 5 and $30 \mathrm{~s}$ were used for the analysis. In the result in Figs. 3 and 4, the successful $60 \mathrm{~s}$ long trials are indicated by solid markers and the $30 \mathrm{~s}$ long trials by empty markers.

\subsection{Stabilometry parameters}

In the literature, postural sway is often analysed using a force distribution measuring plate, which provides the two-dimensional wandering of the CoP in the AP and the ML directions [13, 33-35], which is then used to define stabilometry parameters [36-38]. In case of a uniaxial balance board, oscillations in ML direction are negligible compared to the those in the AP direction, consequently, the balancing task can be described as a one-dimensional motion. Therefore, in this analysis, the angles $\varphi_{a}, \varphi_{h}$ and $\varphi_{b}$ are used to define stabilometry parameters and to characterize improvement of balancing performance.

Nagymáté et al. [36, 37] and Petró et al. [38] collect the most often used stabilometry parameters that can be found in the literature for the characterization of human balancing ability in the AP and the ML directions. Here, we adopt these concepts to the changes in the angles $\varphi_{a}, \varphi_{h}$ and $\varphi_{b}$. Many of the stabilometry parameters for two-dimensional balancing are related and focused on the ratio of changes in the AP and the ML directions, which are not relevant in case of one-dimensional balancing. Here, four different stabilometry parameters are used, three constructed in the time domain and one in the frequency domain. Before calculating the stabilometry parameters, each signal was offset into the mean value.

\subsubsection{Standard Deviation (STD)}

Standard Deviation (STD) is calculated as the square root of the variance and it measures the dispersion of the data relative to its mean value:

$$
\mathrm{STD}=\sqrt{\frac{1}{N-1} \sum_{i=1}^{N}\left|\varphi_{i}-\bar{\varphi}\right|^{2}},
$$

where $N$ is the length of data, $\varphi_{i}$ stands for the corresponding angle at $i^{\text {th }}$ sampling time, and $\bar{\varphi}$ refers to the average of the data.

\subsubsection{Largest Amplitude (LA)}

Considering a signal in time domain, peaks and valleys follow each other. Largest amplitude is defined as the largest peak-valley or valley-peak transition in amplitude

$$
\mathrm{LA}=\max _{i, j}\left(\left|\varphi_{i}-\varphi_{j}\right| \|_{\substack{\operatorname{sgn}\left(\dot{\varphi}_{k}\right)=\mathrm{const} \\ i \leq k \leq j}}\right) .
$$

Calculation of LA was performed using the derivative $\dot{\varphi}$ of the angle signals. As can be seen in Fig. 5, high-frequency noises are superimposed on the signal, which occur as small peaks between the effective peak-valley transitions. In order to remove peaks caused by noise, a moving-average filter was swept with a window length 20 along the corresponding angle signal. After that, boundary of increasing and decreasing parts of the filtered angle data were determined based on the sign of the first derivative of the filtered signal. In the final step, the difference of the adjacent points was calculated and the maximal value was chosen.

\subsubsection{Normalized Path Length (NPL)}

In Nagymáté et al. [37], path length is determined as the length of the total CoP trajectory during the measurement. Adapting this concept to the one-dimensional balancing task, we define the normalized path length as the sum of the absolute value of the angular rotation increment $\varphi_{i+1}-\varphi_{i}$ at each time interval

$\mathrm{NPL}=\frac{1}{t_{b}} \sum_{i=1}^{N-1}\left|\varphi_{i+1}-\varphi_{i}\right|$, 

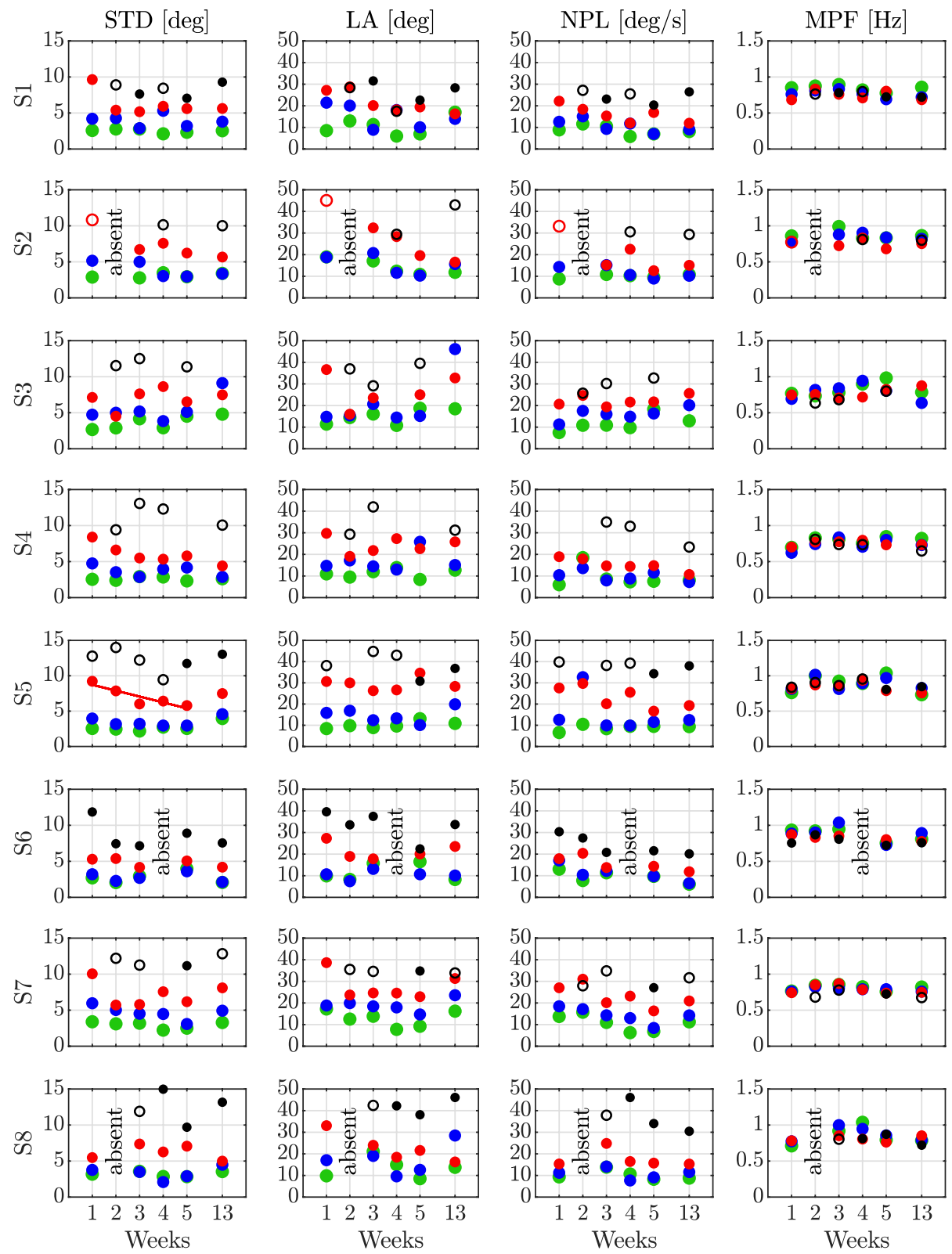

Fig. 3 Stabilometry parameters, experimental results obtained for ankle angle $\varphi_{a}$. Green, blue, red, black markers indicate balance boards of radius $125 \mathrm{~mm}, 100 \mathrm{~mm}, 75 \mathrm{~mm}$ and $50 \mathrm{~mm}$, respectively.

where $t_{b}$ is the balancing time. Due to the normalization by the balancing time, the NPL obtained from the $25 \mathrm{~s}$ data can directly be compared to that obtained from the $50 \mathrm{~s}$ data.

\subsubsection{Mean Power Frequency (MPF)}

Mean power frequency is a weighted average frequency where frequency components $f_{i}$ are weighted by their power $P_{i}$ and $N$ is number of frequency bins:

$$
\text { MPF }=\frac{\sum_{i=1}^{N} f_{i} P_{i}}{\sum_{i=1}^{N} P_{i}} .
$$

Parameters $f_{i}$ and $P_{i}$ were calculated using built-in function of Matlab. Components above $3 \mathrm{~Hz}$ were considered to be noise, therefore data above $3 \mathrm{~Hz}$ were removed as represented in Fig. 5.

\section{Results}

Experiments are evaluated first based on the relevance of the stabilometry parameters, then the development of the balancing skill over the five week learning period is analyzed, finally, the persistence of the balancing skill is verified. 

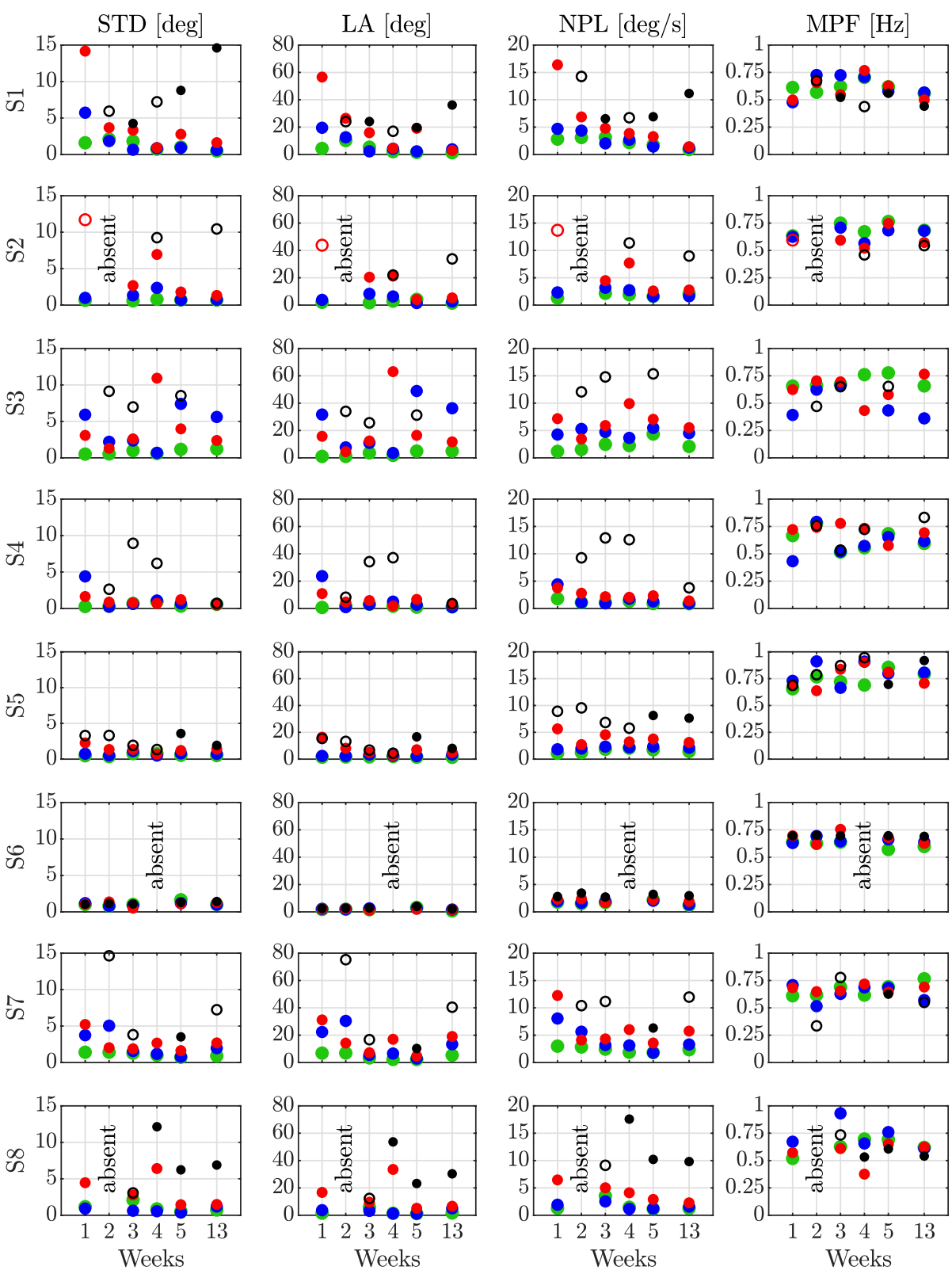

Fig. 4 Stabilometry parameters, experimental results obtained for hip angle $\varphi_{h}$. Green, blue, red, black markers indicate balance boards of radius $125 \mathrm{~mm}, 100 \mathrm{~mm}, 75 \mathrm{~mm}$ and $50 \mathrm{~mm}$, respectively.

\subsection{Analysis of stabilometry parameters}

The above described stabilometry parameters were computed for each balancing trial by each subject and were statistically analyzed over the balancing sessions. The results obtained for ankle angle $\varphi_{a}$ and hip angle $\varphi_{h}$ are shown in Fig. 3 and Fig. 4, respectively. The balance board angle $\varphi_{b}$ was found to be correlated to the ankle angle $\varphi_{a}$ therefore it is not presented in separate figure. Subplots in Fig. 3 and Fig. 4 show the stabilometry parameters associated with the balancing trials. Filled markers correspond to the successful trials of duration $>60 \mathrm{~s}$, while empty markers indicates unsuccessful trials that lasted at least 30 s. Rows of Fig. 3 and Fig. 4 correspond to the participants, and columns are associated with the different stabilometry parameters as indicated at the top of the subplots. The number of balancing sessions (week) is indicated at the bottom subplot. In few cases, some participant could not attend the balancing session due to personal reasons, these experiments are labelled by absent.

All participants were able to stand on the green and blue balance board at least $60 \mathrm{~s}$, as shown by filled markers in Fig. 3 and Fig. 4. Red balance board was a greater 


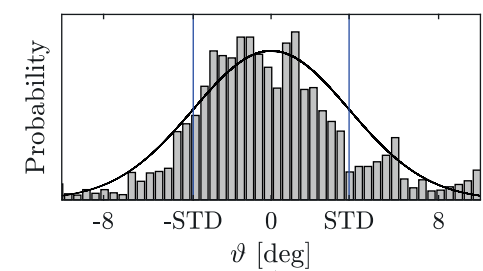

a)

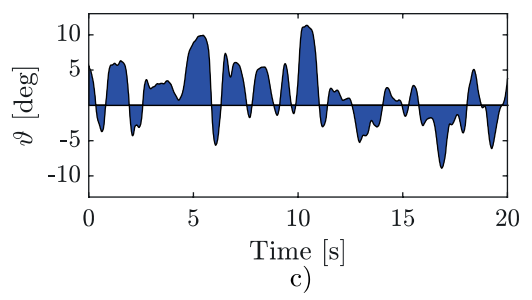

Fig. 5 Stabilometry parameters: a) Standard Deviation (STD) b) Largest Amplitude (LA)

c) Normalized Path Length (NPL) d) Mean Power Frequency (MPF).

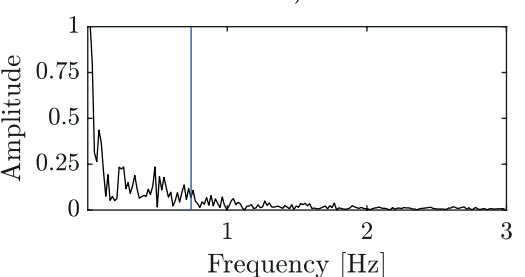

d)

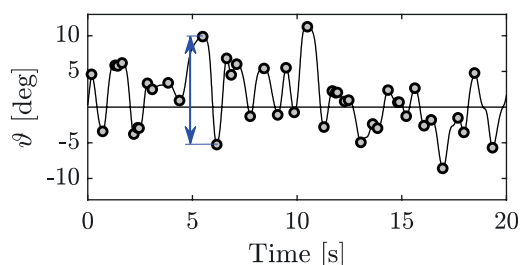

b) challenge, and only five participants were able to stand on the black balance board. S6 was able to stand one-minute-long on all balance board combinations in all balancing sessions. None of the other participants were able to balance on the black balance board on the first week, but four out of them (S1, S5, S7 and S8) were able to balance on it by the fifth week. In general, all the subjects showed some improvement in balancing during the five-week period. Five subjects (S1, S2, S5, S7 and S8) improved by one color level of difficulty, mostly from red to black.

It can be observed that the order of the markers in the STD, LA and NPL plots reflects the difficulty of the balancing task (black $>$ red $>$ blue $>$ green) both in Fig. 3 and Fig. 4 (in some cases the markers are overlapping each other). This indicates that movements at the ankle and the hip gets larger as the task gets more difficult according to the preliminary expectation.

Variation of the MPF over the weeks is not so pronounced as that of STD, LA and NPL. This suggests that MPF is rather related to the subjects balancing style and does not change by practice. The value of MPF is around $0.7-1 \mathrm{~Hz}$ for ankle angle, and a bit lower, 0.5-0.7 Hz for hip angle.

\subsection{Improvement of balancing performance}

Decreasing tendency of the stabilometry parameters are associated with improvement of balancing performance over the five-week period of balancing sessions. In order to identify and quantify this improvement, a straight line was fitted to the stabilometry parameters of each participant for each wheel radius. A sample of the fitted line is shown for S5 with red balance board in Fig. 3. Negative slope indicates decrease in the stabilometry parameter and hence improvement in the balancing performance. The slopes associated with the ankle angle $\varphi_{a}$ are shown in Fig. 6 for all subjects. The mean of slopes is also indicated by empty markers.

One can observe similar tendency of the slopes of STD, LA and NPL in the sense that the mean of slopes is negative (except for the green empty marker in the slope of STD). This indicates that these parameters all reflect the improvement in the balancing abilities over the five weeks of balancing sessions. Correlation between the different stabilometry parameters are shown in Table 1. In contrast, the slope of MPF does not show any tendency related the balancing improvement.

\subsection{Persistence of balancing skill}

Figs. 4 and 5 show the stabilometry parameters measured during the five week learning period and during the confirmation session on the $13^{\text {th }}$ week. The differences between the stabilometry parameters associated with the ankle angle $\varphi_{a}$ on the $1^{\text {st }}$ week and on the $13^{\text {th }}$ week are shown in Fig. 7 for all subjects. The differences are shown only for the green, blue and red balance boards, since nobody except of S6 could stand on the black balance board on the first week. The mean of the differences is also indicated by empty markers. As can be seen, improvement in the STD, LA and NPL values is significant only for the red balance board. For the green and the blue balance board, the mean values of the parameters STD, LA and NPL is about the same on the $1^{\text {st }}$ week and on the $13^{\text {th }}$ week. This indicates that the balancing skill gained during the learning period persisted more for the more difficult task. 

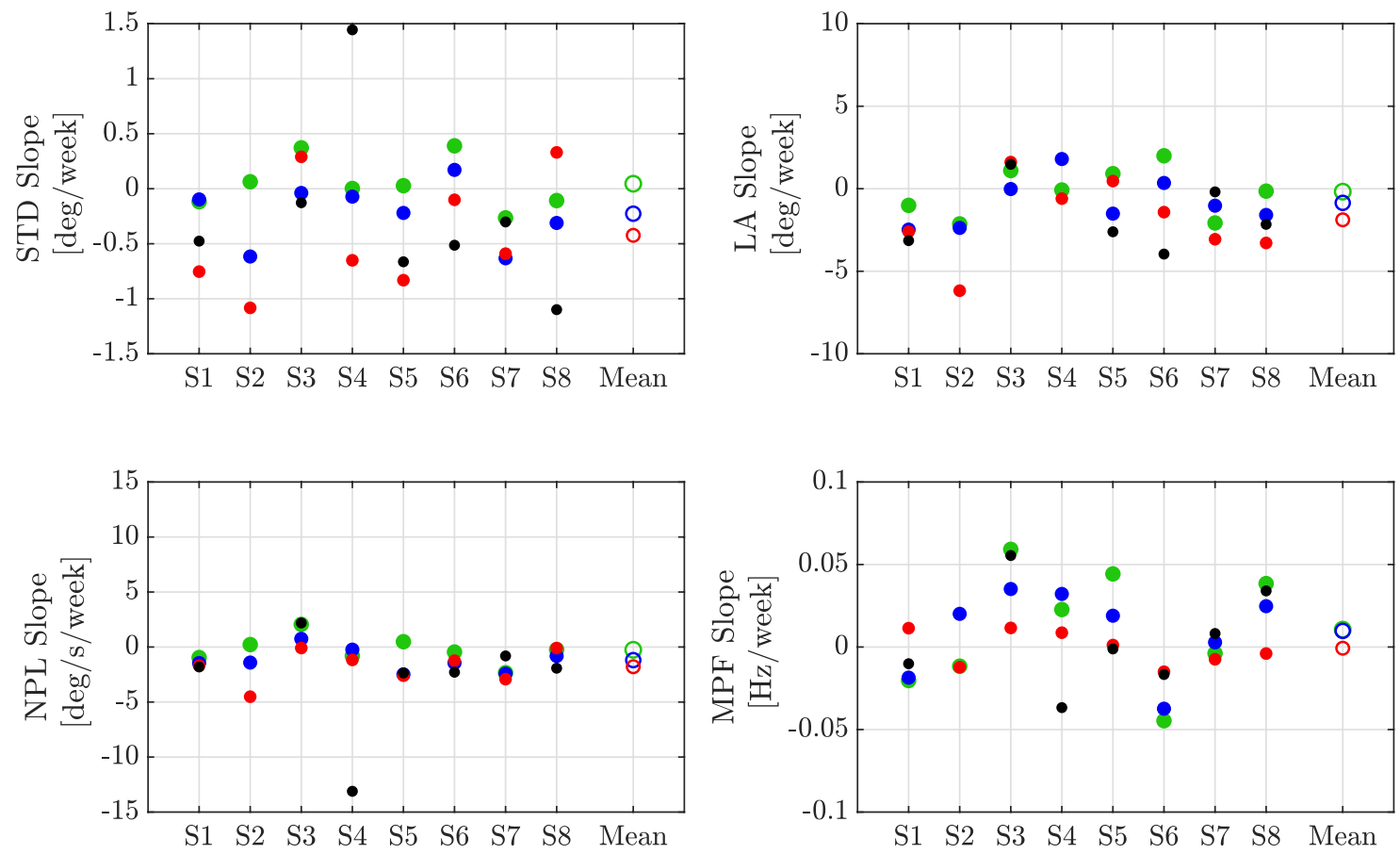

Fig. 6 Slope of the tendency line fitted on the stabilometry parameters for the ankle angle $\varphi_{a}$. Negative slope indicates improvement over the five-week learning period.

Table 1 Correlation of the stabilometry parameters.

\begin{tabular}{lcccc}
\hline \multicolumn{5}{c}{ Correlation coefficient } \\
Stab. par. & STD & LA & NPL & MPF \\
\hline STD & 1 & 0.88 & 0.86 & -0.40 \\
LA & 0.88 & 1 & 0.81 & -0.29 \\
NPL & 0.86 & 0.81 & 1 & -0.10 \\
MPF & -0.40 & -0.29 & -0.10 & 1 \\
\hline
\end{tabular}

\section{Discussion}

Balancing skill is well described by the variation of the ankle angle $\varphi_{a}$ while the variation of the hip angle $\varphi_{h}$ can be used to verify ankle and/or hip strategy. Three out of the four investigated stabilometry parameters were found to reflect improvement in balancing abilities. STD of ankle angle provides an overall picture of improvement of balancing performance. Decreasing tendency of STD parameter means smaller oscillations of the balance board angle around the equilibrium, consequently improving balancing skill. NPL can be interpreted in a similar way: in case of small oscillations, the overall absolute sum of angular deviation is also small. LA is also often used as stabilometry parameter, but it is affected by the momentary concentration of the subjects. If a participant temporary loses concentration, then LA can increase suddenly. Therefore, LA can rather be used to characterize concentration during a balancing trial. MPF is a frequency-based stabilometry parameter, which in this analysis does not show any relation to the improvement of balancing abilities.

\subsection{Ankle and/or hip strategy}

Considering the values of the stabilometry parameters in Fig. 4 and Fig. 5, one can see that oscillation at the ankle is 2-3 times higher than that at the hip. For all subjects, hip STD and NPL is below $5^{\circ}$ in case of green, blue and red balance boards on the last week except for the blue balance board of S3. However, one can observe an extremely large LA parameter of S3 on the last week, which effects STD and NPL, as well. Thus, we can conclude, that ankle strategy is more dominant than hip strategy in case of green, blue, and red balance board, which agrees with the instructions given to the subjects. This indicates that human body for these balance boards can be modelled as a single inverted pendulum indeed.

Five subjects (S1, S5, S6, S7 and S8) were able to balance on the black balance board. For S1, S5, S7 and S8, STD and NPL parameters associated with the black balance board are significantly higher than for the other three balance board settings. This shows that hip strategy becomes important in case of the black balance board (most difficult task) in spite of the instructions given to the subjects that 

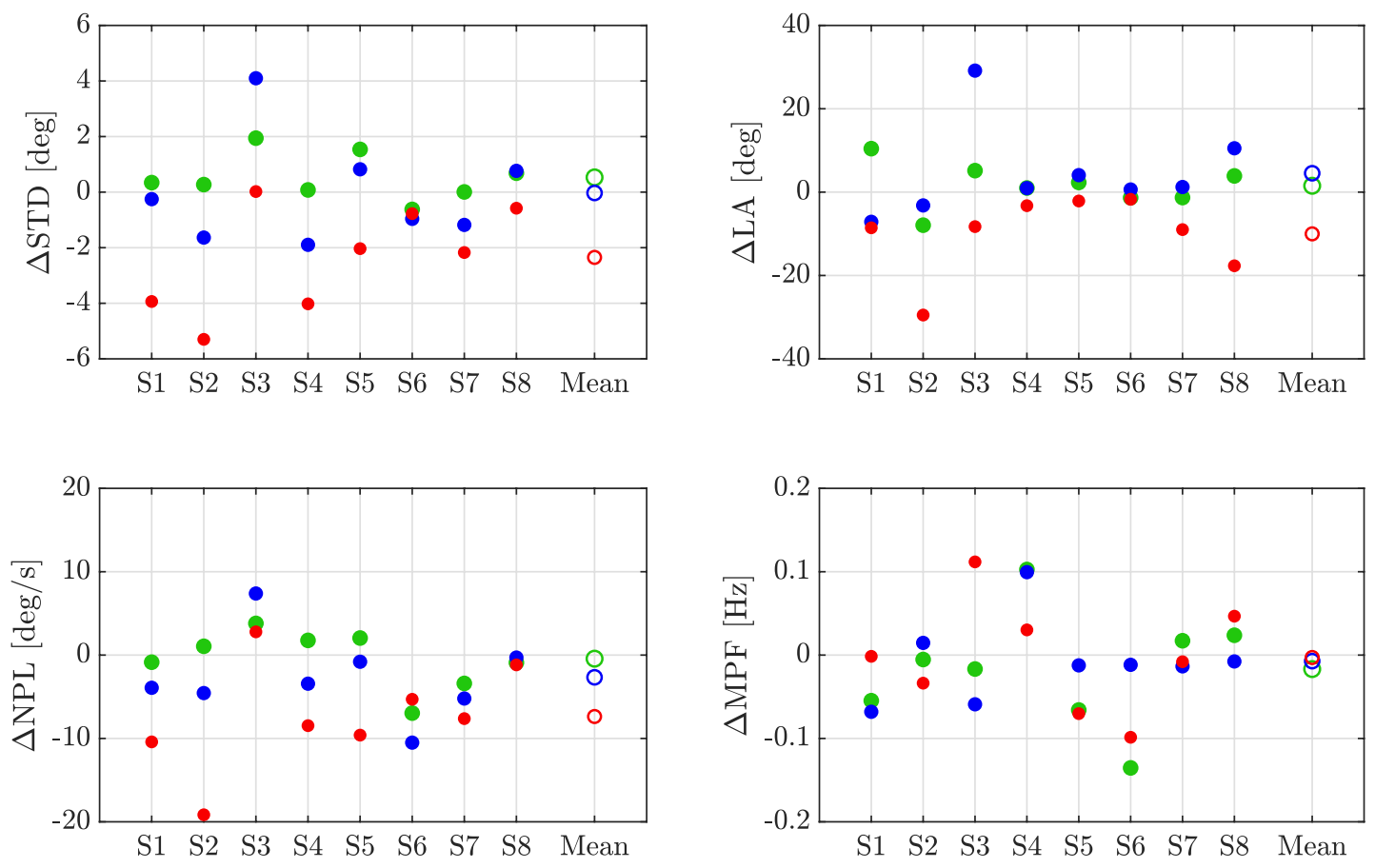

Fig. 7 Difference between the stabilometry parameters on the first balancing session and on the confirmation session.

they shall use only their ankle during the balancing task if possible. The corresponding mechanical model in case of hip strategy is a double inverted pendulum.

Result of S6 obtained for hip angle is different from the other subjects, STD and NPL parameters overlap each other and close to $0^{\circ}$, which indicates that S6 does not employ hip strategy at all.

\subsection{Improvement and persistence of balancing skill}

As explained above, STD and NPL for the ankle angle $\varphi_{a}$ well characterize improvement of balancing skills while LA is also related to temporary loss of concentration. The tendency of STD and NPL is represented by the slope of a line fitted on the data of succeeding balancing sessions. For S1, S2, S4, S5, S6, and S7, the slope of the line fitted to the red and black markers of ankle angle is lower than that of the green and blue markers. This means that these subjects improved better in the more difficult tasks than in the easier tasks. The mean of the slopes obtained for the four balance board setting is shown in the last column of the subplots in Fig. 6 by empty markers. This shows the same tendency: the more difficult the balancing task, the more significant is the improvement.

The balancing session on the $13^{\text {th }}$ week confirmed that balancing abilities gained during the five week learning period persisted for most of the subjects, which is shown by the negative values of the differences $\triangle \mathrm{STD}, \triangle \mathrm{LA}$ and $\triangle N P L$ in Fig. 7. The most dominant improvement was observed for the most difficult task (balancing on the red balance board). This is shown by the negative values of the mean differences $\Delta \mathrm{STD}, \Delta \mathrm{LA}$ and $\Delta \mathrm{NPL}$ in Fig. 7.

\section{Declaration of interest}

We wish to confirm that there are no known conflicts of interest associated with this publication and there has been no significant financial support for this work that could have influenced its outcome.

\section{Acknowledgement}

The research reported in this paper was supported by the Higher Education Excellence Program of the Ministry of Human Capacities in the frame of Biotechnology research area of Budapest University of Technology and Economics (BME FIKP-BIO), by the Hungarian-Chinese Bilateral Scientific and Technological Cooperation Fund under Grant no. 2018-2.1.14-TÉT-CN-2018-00008 and by the UNKP-19-3 New National Excellence Program of the Ministry for Innovation and Technology. 


\section{References}

[1] Abrahamová, D., Hlavacka, F. "Age-related changes of human balance during quiet stance", Physiological Research, 57(6), pp. 957-964, 2008.

[2] Moss, F., Milton, J. G. "Balancing the unbalanced", Nature, 425(6961), pp. 911-912, 2003.

https://doi.org/10.1038/425911a

[3] Robinovitch, S. N., Feldman, F., Yang, Y., Schonnop, R., Leung, P. M., Sarraf, T., Sims-Gould, J., Loughin, M. "Video capture of the circumstances of falls in elderly people residing in long-term care: an observational study", The Lancet, 381(9860), pp. 47-54, 2013.

https://doi.org/10.1016/S0140-6736(12)61263-X

[4] Dionyssiotis, Y. "Analyzing the problem of falls among older people", International Journal of General Medicine, 5, pp. 805-813, 2012.

https://doi.org/10.2147/IJGM.S32651

[5] Young, W. R., Williams, A. M. "How fear of falling can increase fall-risk in older adults: Applying psychological theory to practical observations", Gait \& Posture, 41(1), pp. 7-12, 2015.

https://doi.org/10.1016/j.gaitpost.2014.09.006

[6] Guirguis-Blake, J. M., Michael, Y. L., Perdue, L. A., Coppola, E. L., Beil, T. L. "Interventions to Prevent Falls in Older Adults: Updated Evidence Report and Systematic Review for the US Preventive Services Task Force", JAMA, 319(16), pp. 1705-1716, 2018. https://doi.org/10.1001/jama.2017.21962

[7] Pijnappels, M., Reeves, N. D., Maganaris, C. N., van Dieën, J. H. "Tripping without falling; lower limb strength, a limitation for balance recovery and a target for training in the elderly", Journal of Electromyography and Kinesiology, 18(2), pp. 188-196, 2008. https://doi.org/10.1016/j.jelekin.2007.06.004

[8] Pethes, Á., Bejek, Z., Kiss, R. M. "The effect of knee arthroplasty on balancing ability in response to sudden unidirectional perturbation in the early postoperative period", Journal of Electromyography and Kinesiology, 25(3), pp. 508-514, 2015. https://doi.org/10.1016/j.jelekin.2015.02.010

[9] Cabrera, J. L., Milton, J. G. "On-Off Intermittency in a Human Balancing Task", Physical Review Letters, 89(15), Article Number: 158702, 2002. https://doi.org/10.1103/PhysRevLett.89.158702

[10] Mehta, B., Schaal, S. "Forward Models in Visuomotor Control", Journal of Neurophysiology, 88(2), pp. 942-953, 2002. https://doi.org/10.1152/jn.2002.88.2.942

[11] Nagy, D. J., Bencsik, L., Insperger, T. "Experimental estimation of tactile reaction delay during stick balancing using cepstral analysis", Mechanical Systems and Signal Processing, 138, Article Number: 106554, 2020. https://doi.org/10.1016/j.ymssp.2019.106554

[12] Maurer, C., Peterka, R. J. "A New Interpretation of Spontaneous Sway Measures Based on a Simple Model of Human Postural Control", Journal of Neurophysiology, 93(1), pp. 189-200, 2005. https://doi.org/10.1152/jn.00221.2004

[13] Kiemel, T., Zhang, Y., Jeka, J. J. "Identification of Neural Feedback for Upright Stance in Humans: Stabilization rather than Sway Minimization", The Journal of Neuroscience, 31(42), pp. 15144-15153, 2011.

https://doi.org/10.1523/JNEUROSCI.1013-11.2011
[14] Nomura, T., Oshikawa, S., Suzuki, Y., Kiyono, K., Morasso, P. "Modeling human postural sway using an intermittent control and hemodynamic perturbations", Mathematical Biosciences, 245(1), pp. 86-95, 2013.

https://doi.org/10.1016/j.mbs.2013.02.002

[15] Pasma, J. H., Boonstra, T. A., van Kordelaar, J., Spyropoulou, V. V., Schouten, A. C. "A Sensitivity Analysis of an Inverted Pendulum Balance Control Model", Frontiers in Computational Neuroscience, 11, Article Number: 99, 2017. https://doi.org/10.3389/fncom.2017.00099

[16] Buza, G., Milton, J., Bencsik, L., Insperger, T. "Establishing metrics and control laws for the learning process: ball and beam balancing", Biological Cybernetics, 114(1), pp. 83-93, 2020. https://doi.org/10.1007/s00422-020-00815-Z

[17] Li, Y., Levine, W. S., Loeb, G. E. "A Two-Joint Human Posture Control Model With Realistic Neural Delays", IEEE Transactions on Neural Systems and Rehabilitation Engineering, 20(5), pp. 738-748, 2012. https://doi.org/10.1109/TNSRE.2012.2199333

[18] Suzuki, Y., Nomura, T., Casadio, M., Morasso, P. "Intermittent control with ankle, hip, and mixed strategies during quiet standing: a theoretical proposal based on a double inverted pendulum model", Journal of Theoretical Biology, 310, pp. 55-79, 2012. https://doi.org/10.1016/j.jtbi.2012.06.019

[19] Morasso, P., Cherif, A., Zenzeri, J. "Quiet standing: The Single Inverted Pendulum model is not so bad after all", PLoS ONE 14(3), Article Number: e0213870, 2019.

https://doi.org/10.1371/journal.pone.0213870

[20] Chagdes, J. R., Rietdyk, S., Haddad, J. M., Zelaznik, H. N., Raman, A. "Dynamic stability of a human standing on a balance board", Journal of Biomechanics, 46(15), pp. 2593-2602, 2013. https://doi.org/10.1016/j.jbiomech.2013.08.012

[21] Cruise, D. R., Chagdes, J. R., Liddy, J. J., Rietdyk, S., Haddad, J. M., Zelaznik, H. N., Raman, A. "An active balance board system with real-time control of stiffness and time delay to assess mechanisms of postural stability", Journal of Biomechanics, 60, pp. 48-56, 2017. https://doi.org/10.1016/j.jbiomech.2017.06.018

[22] Chumacero-Polanco, E., Yang, J. "Basin of Attraction and Limit Cycle Oscillation Amplitude of an Ankle-Hip Model of Balance on a Balance Board", Journal of Biomechanical Engineering, 141(11), Article Number: 111007, 2019. https://doi.org/10.1115/1.4043563

[23] Asai, Y., Tasaka, Y., Nomura, K., Nomura, T., Casadio, M., Morasso, P. "A Model of Postural Control in Quiet Standing: Robust Compensation of Delay-Induced Instability Using Intermittent Activation of Feedback Control", PLoS ONE, 4(7), Article Number: e6169, 2009. https://doi.org/10.1371/journal.pone.0006169

[24] Gawthrop, P., Loram, I., Gollee, H., Lakie, M. "Intermittent control models of human standing: similarities and differences", Biological Cybernetics, 108(2), pp. 159-168, 2014. https://doi.org/10.1007/s00422-014-0587-5 
[25] Stepan, G. "Delay effects in the human sensory system during balancing", Philosophical Transactions of the Royal Society A: Mathematical, Physical and Engineering Sciences, 367(1891), pp. 1195-1212, 2009.

https://doi.org/10.1098/rsta.2008.0278

[26] Milton, J., Meyer, R., Zhvanetsky, M., Ridge, S., Insperger, T. "Control at stability's edge minimizes energetic costs: expert stick balancing", Journal of The Royal Society Interface, 13(119), Article Number: 20160212, 2016. https://doi.org/10.1098/rsif.2016.0212

[27] Zana, R. R., Zelei, A. "Introduction of a Complex Reaction Time Tester Instrument", Periodica Polytechnica Mechanical Engineering, 64(1), pp. 20-30, 2020. https://doi.org/10.3311/PPme.13807

[28] Herold, F., Orlowski, K., Börmel, S., Müller, N. G. "Cortical activation during balancing on a balance board", Human Movement Science, 51, pp. 51-58, 2017. https://doi.org/10.1016/j.humov.2016.11.002

[29] Molnar, Cs. A., Zelei, A., Insperger, T. "Human balancing on rolling balance board in the frontal plane", IFAC-PapersOnLine, 51(14), pp. 300-305, 2018.

https://doi.org/10.1016/j.ifacol.2018.07.240

[30] Chumacero-Polanco, E., Yang, J. "Effect of disturbances and sensorimotor deficits on the postural robustness of an ankle-hip model of balance on a balance board", Nonlinear Dynamics, 99(3), pp. 1959-1973, 2020.

https://doi.org/10.1007/s11071-019-05403-w

[31] Molnar, Cs. A., Zelei, A., Insperger, T. "Estimation of human reaction time delay during balancing on balance board", In: 2017 13th IASTED International Conference on Biomedical Engineering (BioMed), Innsbruck, Austria, 2017, pp. 176-180. https://doi.org/10.2316/P.2017.852-048
[32] Stewart, J. M., Clarke, D. "'He's Dizzy When He Stands Up": An Introduction to Initial Orthostatic Hypotension", The Journal of Pediatrics, 158(3), pp. 499-504, 2011.

https://doi.org/10.1016/j.jpeds.2010.09.004

[33] Bartlett, H. L., Ting, L. H., Bingham, J. T. "Accuracy of force and center of pressure measures of the Wii Balance Board", Gait \& Posture, 39(1), pp. 224-228, 2014.

https://doi.org/10.1016/j.gaitpost.2013.07.010

[34] Tanabe, H., Fujii, K., Kouzaki, M. "Large postural fluctuations but unchanged postural sway dynamics during tiptoe standing compared to quiet standing", Journal of Electromyography and Kinesiology, 22(6), pp. 975-982, 2012. https://doi.org/10.1016/j.jelekin.2012.05.006

[35] Piras, A., Raffi, M., Perazzolo, M., Squatrito, S. "Influence of heading perception in the control of posture", Journal of Electromyography and Kinesiology, 39, pp. 89-94, 2018. https://doi.org/10.1016/j.jelekin.2018.02.001

[36] Nagymáté, G., Kiss, R. M. "Parameter Reduction in the Frequency Analysis of Center of Pressure in Stabilometry", Periodica Polytechnica Mechanical Engineering, 60(4), pp. 238-246, 2016. https://doi.org/10.3311/PPme.8999

[37] Nagymáté, G., Orlovits, Zs., Kiss, R. M. "Reliability analysis of a sensitive and independent stabilometry parameter set", PLoS ONE, 13(4), Article Number: e0195995, 2018. https://doi.org/10.1371/journal.pone.0195995

[38] Petró, B., Papachatzopoulou, A., Kiss, R. M. "Devices and tasks involved in the objective assessment of standing dynamic balancing - A systematic literature review", PLoS ONE, 12(9), Article Number: e0185188, 2017

https://doi.org/10.1371/journal.pone.0185188 